\title{
Infecção de sítio cirúrgico no seguimento pós-alta: impacto na incidência e avaliação dos métodos utilizados
}

\author{
INFECTION OF SURGICAL SITE IN THE FOLLOWING POSTDISCHARGE: \\ IMPACT IN THE INCIDENCE AND EVALUATION OF THE USED METHODS \\ INFECCIÓNDELSITIOQUIRÚRGICOENEL SEGUIMIENTODEPUÉSDELALTAHOSPITALARIO: \\ IMPACTO DE LAINCIDENCIAY EVALUACIÓN DE LOS MÉTODOS UTILIZADOS
}

\section{Adriana Cristina Oliveira1', Suely Itsuko Ciosak ${ }^{2}$}

\section{RESUMO}

Trata-se de um estudo prospectivo realizado em dois hospitais de ensino. Foram acompanhados 501 pacientes de agosto de 2001 a março de 2002, submetidos à cirurgia do aparelho digestivo, sendo diagnosticadas 140 infecções do sítio cirúrgico (ISC). 31 ISC intrahospitalares e 109 após a alta. A incidência da ISC intrahospitalar foi de $6,2 \%$, elevando-se para $28,0 \%$ com a vigilância pós-alta. Os métodos de vigilância pós-alta são discutidos e dentre as várias opções não há uma recomendada como a melhor. Sugere-se, portanto, que algum tipo de vigilância após a alta seja realizada, mas a escolha do método dependerá dos recursos de cada instituição.

\section{DESCRITORES}

Infecção da ferida operatória.

Vigilância (métodos).

\begin{abstract}
It is a prospective study in two teaching hospitals. They accompanied 501 admitted patients who were submitted to the surgery of the digestive system from August 2001 to March 2002. In the period of the study, 140 surgical site infections (SSIs) were diagnosed. Thirty-one SSIs were diagnosed in the hospital and 109 after the discharge. The incidence of SSIs was of $6,2 \%$, rising for $28,0 \%$, with postdischarge surveillance. The methods of postdischarge surveillance are also discussed and among the several options, as there is not one recommended as the best. However, it is suggested that some types of postdischarge surveillance must be accomplished. The choice of the method will depend on the sources of each institution.
\end{abstract}

\section{DESCRIPTORS}

Surgical wound infection.

Surveillance (methods)

\section{RESUMEN}

Se trata de un estudio prospectivo realizado en dos hospitales de enseñanza. Fueron acompañados 501 pacientes de agosto de 2001 a marzo del 2002, sometidos a cirugía del aparato digestivo, siendo diagnosticadas 140 infecciones del sitio quirúrgico (ISQ). Del total 31 ISQ fueron intra hospitalarias y 109 después del alta. La incidencia de las ISQ intra-hospitalarias fue de $6.2 \%$ elevándose a $28.0 \%$ con la vigilancia después del alta. Los métodos de vigilancia post- alta son discutidos y entre las varias opciones no hay ninguna recomendada como la mejor. Se sugiere, por lo tanto, que algún tipo de vigilancia después del alta sea realizada, mas la elección del método dependerá de los recursos de cada institución.

\section{DESCRIPTORES}

Infección de herida operatoria. Vigilancia (metodos)
1 Professora Doutora do Departamento de Enfermagem Básica da Escola de Enfermagem da Universidade Federal de Minas Gerais (UFMG) acoliveira@enf.ufmg.br 2 Professora Doutora do Departamento de Enfermagem em Saúde Coletiva da Escola de Enfermagem da Universidade de São Paulo (EEUSP). siciosak@usp.br 


\section{INTRODUÇÃO}

Dentre as infecções hospitalares, a infecção do sitio cirúrgico (ISC) é a segunda mais importante entre os pacientes hospitalizados, sendo suplantada somente pela infecção urinária. No entanto, em diversas instituições a ISC ainda ocupa o primeiro lugar, sendo a infecção mais prevalente ${ }^{(1-3)}$.

Estima-se que no Brasil, a ISC apresente uma incidência de 2,8 a 20\%, (média de 11\%) dependendo do tipo de vigilância realizada, das características do hospital, do paciente e do procedimento cirúrgico ${ }^{(4)}$. Estudos revelam que a ocorrência da ISC pode elevar em média a permanência hospitalar de 7,4 para $14,3 \operatorname{dias}^{(2-3,5)}$.

A ISC, especialmente aquela relacionada à órgãos ou cavidades profundas, é importante causa de morbi-letalidade e da variação do custo do tratamento relacionado à necessidade da terapia antimicrobiana, ocasionais reintervenções cirúrgicas com aumento do tempo de permanência e ainda a possibilidade de exposição a patógenos multirresistentes ${ }^{(1-6)}$.

A ocorrência da ISC não deve ser considerada apenas no período de hospitalização, segundo a vigilância do paciente cirúrgico, proposta pelo Centro de Controle de Doenças de Atlanta, nos Estados Unidos, (CDC) em 1992. Os pacientes cirúrgicos devem ser acompanhados desde a cirurgia até a alta hospitalar e seguidos após a alta hospitalar, pelo período de até trinta dias da data da cirurgia ou em caso de implante de prótese até um ano $o^{(5,7)}$

Entretanto, muitas vezes a vigilância epidemiológica dos pacientes cirúrgicos só ocorre durante o período de internação e a incidência da ISC não inclui as infecções apresentadas pelo paciente após a alta hospitalar, devido a não realização de controle pós-alta.

O Serviço de Controle de Infecção Hospitalar (SCIH) que não possui controle de egresso cirúrgico gera taxa subnotificada ${ }^{(3,7)}$. E, para o CDC a incidência de ISC após a alta hospitalar pode variar entre 12 a $84 \%{ }^{(1)}$.

A detecção da ISC após a alta hospitalar é uma forma essencial para obtenção de taxas acuradas, além disso, a Joint Commission on Accreditation of Healthcare Organization
(JCAHO) considera que fazer a vigilância pósalta, entre outras coisas, é imprescindível por permitir comparações inter-hospitalares ${ }^{(8)}$.

Os métodos de seguimento do paciente após a alta, mais utilizados atualmente, são: retorno ambulatorial, contato telefônico, carta-questionário, divergindo entre si a indicação e a acurácia de cada um deles ${ }^{(1,3,7-9)}$.

Reconhecendo a relevância do tema e a participação direta do enfermeiro no processo de vigilância epidemiológica das infecções hospitalares, este estudo teve por objetivos determinar a incidência da ISC nos pacientes submetidos à Cirurgia do Aparelho Digestivo (CAD) durante a hospitalização e no acompanhamento em Ambulatório de Egressos; verificar o impacto da sub-notificação da ISC após a alta hospitalar e discutir os métodos de vigilância utilizados.

\section{METODOLOGIA}

Tratou-se de um estudo epidemiológico, descritivo, tipo coorte e prospectivo, realizado em dois hospitais de grande porte, de cuidados terciários localizados na cidade de São Paulo.

Para a inclusão dos pacientes no estudo, foi utilizada a recomendação do National Nosocomial Infection Surveillance (NISS) ${ }^{(7)}$. Assim, foram acompanhados todos os pacientes da CAD admitidos no período de agosto de 2001 à março de 2002, durante a internação e após a alta hospitalar, que atendiam aos seguintes critérios $^{(7)}$ : ser admitido para cirurgia do aparelho digestivo, permanecer hospitalizado por mais de 24 horas e realizar um procedimento cirúrgico que incluísse incisão e sutura, antes do paciente deixar o centro cirúrgico.

Cabe ressaltar que foram acompanhados os pacientes cirúrgicos, somente, para diagnóstico da infecção do sítio cirúrgico, não tendo sido aqui referido, outras infecções, por não constituírem objetivo deste estudo.

Após a admissão, todos os pacientes cirúrgicos foram acompanhados diariamente, até a alta hospitalar. Os dados foram coletados durante a internação através de busca ativa nos prontuários dos pacientes, registros médicos e de enfermagem e, algumas vezes, por visitas para avaliação da incisão cirúrgica, em relação à presença de infecção. 
Para o diagnóstico da ISC, conforme a metodologia utilizada (NNIS), considerouse como padrão-ouro a presença de secreção purulenta (com ou sem confirmação laboratorial) desde que a secreção purulenta não caracterizasse reação local ao ponto.

A ISC foi classificada como: superficial (se localizada acima da fáscia muscular), profunda (se localizada entre a fáscia e a camada muscular) e órgão/cavidade (quando envolvesse estruturas anatômicas, abertas ou manipuladas, durante o ato cirúrgico $)^{(5,7)}$.

A vigilância pós-alta, do paciente cirúrgico, foi realizada por meio do retorno ambulatorial e de contato telefônico. Os pacientes foram avaliados em torno de sete a dez dias, após a data da cirurgia, no retorno ambulatorial para retirada de pontos e, posteriormente, o segundo contato foi realizado por telefone, no período de 14 a 30 dias também em relação à data da cirurgia.

O contato ambulatorial foi realizado de acordo com a data agendada para o controle médico (retirada de pontos/revisão da cirurgia) a fim de evitar ao paciente custos adicionais com transporte. Neste retorno, o paciente foi avaliado em momentos distintos, pela equipe médica e pela equipe do estudo, sendo que, em caso de dúvidas, as equipes discutiam o caso específico.
Depois de coletados, em instrumentos específicos, os dados foram codificados para as diversas variáveis do estudo e digitados em banco de dados destinado à este fim, em ACESS, sendo posteriormente utilizado para a análise estatística juntamente com o SPSS, a partir de freqüência simples, absoluta e percentual.

\section{RESULTADOS E DISCUSSÃO}

A amostra foi composta por 501 pacientes. Durante o período do estudo foram diagnosticadas 140 ISC, com uma incidência global de $28,0 \%$, sendo que 31 destas ISC (31/140) foram diagnosticadas no período intra-hospitalar $(22,1 \%)$ e 109 foram diagnosticadas após a alta (77,9\%), conforme apresentado na Tabela 1. Desta forma, pode-se constatar que o impacto da incidência da ISC com o controle pós-alta foi de $21,8 \%$.

Este achado traduz o que ocorre na maioria dos serviço de controle de infecção hospitalar que não realizam a vigilância pós-alta do paciente cirúrgico, pois o diagnóstico de apenas 31 ISC durante o período de inter-nação fornece um falso parâmetro de incidência de $6,2 \%$, onde o resultado obtido denota uma pseudo tranqüilidade em relação a incidência real da ISC, impedindo que medidas de prevenção e controle sejam adotadas de forma eficiente e eficaz.

Tabela 1 - Distribuição da infecção do sítio cirúrgico (ISC) segundo o momento do diagnóstico e a incidência. (São Paulo, 2002)

\begin{tabular}{l|c|c|c}
\hline \multirow{2}{*}{$\begin{array}{c}\text { Momento do } \\
\text { diagnóstico }\end{array}$} & Sim & ISC & Taxa ISC* \\
\cline { 2 - 4 } & 31 & 22,1 & $\%$ \\
\hline Intra-hospitalar & 109 & 77,9 & 6,2 \\
Após a alta & 140 & 100,0 & 21,8 \\
\hline TOTAL & &
\end{tabular}

Dentre as 140 ISC diagnosticadas no período do estudo, 127 (90,7\%) foram classififundas e 3 (2,1\%) como de órgão/cavidade, conforme apresentado na Tabela 2. cadas como superficiais, $10(7,2 \%)$ foram pro-

Tabela 2- Distribuição da infecção do sítio cirúrgico (ISC) segundo a classificação da ISC e o momento diagnóstico. (São Paulo, 2002)

\begin{tabular}{l|c|c|c|c|c|c}
\hline \multirow{2}{*}{$\begin{array}{c}\text { Classificação } \\
\text { da ISC }\end{array}$} & \multicolumn{3}{|c|}{ ISC } & \multicolumn{2}{c}{ Total } \\
\cline { 2 - 7 } & \multicolumn{2}{|c|}{ Intra-hospitalar } & \multicolumn{2}{c}{ Pós-alta } & \multicolumn{2}{c}{$\mathbf{1 0 0 , 0 \%}$} \\
\cline { 2 - 7 } & $\mathbf{N}$ & $\%$ & $\mathbf{N}$ & $\%$ & $\mathbf{N}$ & $\%$ \\
\hline Superficial & 22 & $(17,3)$ & 105 & $(82,7)$ & 127 & $(100,0)$ \\
Profunda & 8 & $(80,0)$ & 2 & $(20,0)$ & 10 & $(100,0)$ \\
Órgão/ cavidade & 1 & $(33,3)$ & 2 & $(66,4)$ & 3 & $(100,0)$ \\
\hline TOTAL & 31 & $(22,1)$ & 109 & $(77,9)$ & 140 & $(100,0)$ \\
\hline
\end{tabular}


Adriana Cristina Oliveira Suely Itsuko Ciosak
Pode-se afirmar que a infecção da ferida cirúrgica é um risco inerente ao ato cirúrgico, sendo a complicação mais freqüente, que pode manifestar-se pela presença de eritema local, edema, calor, dor deiscência e secreção purulenta. Porém, pela subjetividade/dificuldade na interpretação dos sinais de inflamação e, também pelo fato de que estes podem estar atribuídos a outro evento que não especificamente a ISC, é que se aceita a definição de infecção da ferida cirúrgica como aquela que apresenta drenagem de secreção purulenta isoladamente, desde que não restrita ao ponto, com ou sem confirmação laboratorial ${ }^{(5,10-11)}$. Este critério, constitui algumas vezes, uma questão de divergência entre o diagnóstico da ISC pela equipe médica e a do controle de infecção, fato este que reforça a ocorrência da subnotificação pós-alta pelo cirurgiões.

Segundo o intervalo do diagnóstico da ISC intra-hospitalar e após a alta, verificou-se que no período de 0 à $<7$ dias foram diagnosticados 50 ISC (37,5\%), no período $\geq 7$ à $<14$ dias $56(40,0 \%)$, no período $\geq 14$ à $<21$ dias $18(12,9 \%)$ e no período $\geq 21$ dias $16(11,5 \%)$.

Assim sendo, verifica-se que $90,4 \%$ das ISC se manifestaram até o vigésimo-primeiro dia da data da cirurgia.

Dados como o intervalo em dias, entre a data da cirurgia e a ocorrência da ISC, constituem de importância fundamental para que cada instituição estabeleça seu período ideal para o seguimento do paciente cirúrgico, tendo em vista que o período de trinta dias é muitas vezes controverso para alguns autores $^{(5,12)}$.

Por outro lado, a realização da vigilância pós-alta apesar de ser importante como parte da vigilância do paciente cirúrgico, nem sempre é tão fácil de ser implementada, considerando que a escolha do método depende de: estrutura da instituição, tipo de hospital, tipo de clientela atendida, infra-estrutura e recursos humanos disponíveis.

Alguns pontos merecem destaque sobre a avaliação dos métodos de vigilância pósalta descritos na literatura e a experiência vivenciada neste estudo. Verifica-se, na prática, que a implementação do controle pósalta, através de ambulatório de retorno (ou de egresso) do paciente cirúrgico, na maioria das vezes, só se torna possível e concreta em hospitais públicos e/ou universitários, onde a clientela atendida retorna à mesma instituição, para a retirada dos pontos e controle médico da cirurgia.

Um dos serviços de referência, no Brasil, para o seguimento do paciente cirúrgico após a alta hospitalar, em ambulatório, é o serviço do Hospital das Clínicas da Universidade Federal de Pernambuco (HC/UFPE), que realiza este serviço desde $1979^{(12)}$. Há ainda, o ambulatório de egressos cirúrgicos, do Hospital das Clínicas da Universidade Federal de Minas Gerais, que funciona desde $1998^{(9)}$.

$\mathrm{O}$ aspecto positivo deste tipo de vigilância, por ambulatório, além da busca ativa dos casos, é a consistência dos dados obtidos. Sendo o avaliador um profissional do Serviço de Controle de Infecção Hospitalar (SCIH), a confiabilidade do serviço consitui outro fator de extrema importância, no momento de retorno das taxas ao cirurgião, que pode ser considerada pela padronização de critérios diagnósticos utilizados intra-hospitalar e após a alta, bem como, a capacitação e experiência do profissional.

A mesma facilidade para a implementação do serviço de vigilância do paciente cirúrgico, por ambulatório de egresso, nem sempre é conseguida em instituições privadas, onde o retorno do paciente geralmente ocorre em consultórios particulares, impedindo que os esforços da SCIH possam alcançar a notificação dos pacientes com ISC após a alta.

O uso de métodos como carta-questionários aos cirurgiões, pacientes ou a ambos, nem sempre é confiável, pois o retorno destas, em geral é baixo, apesar do envio de cartas seladas sem nenhum custo para seu retorno. Por outro lado, há que se considerar, também, que a homogeneidade de critérios utilizados para o diagnóstico da ISC, como já apontado, freqüentemente, divergem entre a equipe médica e a equipe do SCIH.

O SCIH geralmente adota os critérios padronizados pelo $\mathrm{CDC}$, onde o padrão-ouro para a identificação da ISC é a presença da secreção purulenta ${ }^{(1,5)}$. Em contra partida, verifica-se que para o cirurgião, na maioria das vezes, a presença de secreção purulenta, (não restrita ao ponto), nem sempre significa a necessidade de notificação da ISC. Para ele, a secreção purulenta é geralmente produto 
de drenagem espontânea, sendo algumas vezes indicado apenas o uso de calor local, sem nenhuma necessidade do uso de agentes antimicrobianos. Porém, esta é a ISC que mais freqüentemente ocorre após a alta hospitalar, ou seja, infecções que se localizam superficialmente acima do tecido celular subcutâneo $^{(1,5,8-9)}$.

Constata-se, portanto, que a vigilância pósalta deve ser realizada por um profissional treinado segundo os critérios diagnósticos utilizados pelo SCIH, de forma a manter a homogeneidade da conduta diante da notificação da ISC, durante a internação e após a alta.

Além disso, observa-se na literatura alguns estudos que enviam junto à carta-questionário aos cirurgiões, os critérios de notificação utilizados pelo SCIH. Nesta situação, há que se destacar a importância de um trabalho prévio, a fim de despertar a motivação e o interesse da equipe cirúrgica em participar e colaborar com o SCIH, pois, a maioria destes estudos, ainda, mostram uma baixa adesão da equipe cirúrgica no retorno dos questionários $^{(1,8)}$.

Um fato negativo, que também merece destaque, é a rotatividade da equipe médica, quando se considera a sua participação neste tipo de vigilância em hospitais públicos e/ ou universitários, onde existam programas de treinamentos em residência médica.

Outro método de vigilância pós-alta, refere-se à monitorização/vigilância por telefone. Neste tipo de vigilância o contato é direcionado ao paciente, após sua alta, entre o sétimo e décimo dia e, um segundo contato entre o décimo-quarto e trigésimo dia da data da cirurgia.

Para a garantia do registro correto do número do telefone, se possível, recomenda-se que o mesmo seja checado individualmente com cada paciente, a fim de se estabelecer um contato inicial de identificação do SCIH e destacar o acompanhamento que será realizado após sua alta, obtendo o seu consentimento para tal.

Desta forma, o contato telefônico necessita ser realizado de forma padronizada, através de um impresso previamente testado e validado pelo $\mathrm{SCIH}$, onde as perguntas sejam direcionadas ao paciente de forma objetiva, não induzindo a respostas que levem a supernotificação da ISC.
É importante levar em conta que mesmo que, mesmo que o paciente apresente ISC no primeiro contato, a realização do segundo contato é imprescindível, para confirmação de que a ISC se manteve no mesmo sítio especifico, notificado anteriormente (superficial, profunda ou de órgão/cavidade), além de se obter um melhor controle da evolução da mesma.

Algumas dificuldades a serem evitadas com o uso da vigilância por telefone, referemse ao agendamento de cada paciente, sempre agrupando o maior número possível de pacientes para contato no mesmo dia, considerando o intervalo entre a cirurgia e o período de retirada de pontos (padronizada pelo serviço de cirurgia da instituição), além de um segundo controle após o período destinado pela equipe cirúrgica para a revisão da cirurgia realizada.

As ligações telefônicas devem ter, preferencialmente, dia fixo para sua realização impedindo o excessivo desgaste da equipe do SCIH com a realização deste tipo de vigilância de forma desordenada, o que poderá ocasionar uma sobrecarga de trabalho, refletindo no desânimo para a continuidade da vigilância.

Assim sendo, os contatos telefônicos devem ser planejados, organizados por pacientes, com a disponibilidade dos impressos utilizados durante a internação, de forma que o profissional possa ter em mãos, no momento do contato todo o histórico de internação do paciente como: nome completo, data da admissão, data da cirurgia, tipo de cirurgia realizada, equipe médica que a realizou e se houve ou não o diagnóstico da ISC durante a internação, evitando assim, o consumo excessivo do tempo nas ligações e também questionamentos desnecessários ao paciente, que possam ser interpretados como uma baixa credibilidade no SCIH e na instituição.

Não obstante, dos diversos métodos de vigilância pós-alta discutidos neste estudo, foram utilizados a vigilância em ambulatório associada a vigilância por contato telefônico. Pode-se afirmar que a abrangência do retorno das informações (por retorno ambulatorial e contato telefônico) foi satisfatória, com um índice de perda inferior a $10 \%$. Tais perdas foram associadas a um pequeno número de pacientes que não possuíam telefone, alguns telefones que foram
Infecção de sítio cirúrgico no seguimento pós-alta: impacto na incidência e avaliação dos métodos utilizados 
Adriana Cristina Oliveira Suely Itsuko Ciosak fornecidos incorretamente e outros que estavam programados para não receber ligações.

Os dados do nosso estudo apresentam melhores resultados que os encontrados em $1995^{(12)}$, no qual a variação do retorno ambulatorial do paciente de $64 \%$ a $84 \%$, foi considerado como satisfatório. Este diferencial pode ser atribuído ao seguimento misto que adotamos, ou seja, realizado através do ambulatório e do contato telefônico.

Adicionalmente, este tipo de vigilância também possui, um lado de marketing institucional, que pode influenciar na ampliação da credibilidade do serviço prestado, permitindo ao paciente interpretar que a preocupação com a vigilância pós-alta não se baseia na "alta taxa de ISC da instituição", mas sim por representar um serviço de melhoria contínua da assistência prestada em todos os níveis, funcionando como um controle de qualidade do serviço prestado pela instituição.

A incidência de ISC com a vigilância após a alta hospitalar é fundamental nos serviços de saúde pois, traduz um dos indicadores da qualidade da assistência prestada numa instituição, requerida sempre e principalmente para a acreditação hospitalar, além da fiscalização pelos serviços de vigilância epidemiológica regional, estadual e federal.

\section{CONCLUSÕES}

Por meio deste estudo, foi possível verificar que o impacto da sub-notificação da ISC foi de $21,8 \%$, sendo que $77,9 \%$ das ISC não teriam sido notificadas sem a realização do seguimento pós-alta, considerando que a in- cidência da ISC, obtida apenas durante o período de internação, foi de $6,2 \%(31 / 501)$.

Em relação à avaliação dos métodos de vigilância após a alta hospitalar do paciente cirúrgico, considera-se que dentre as várias opções comentadas no estudo, não há uma delas que possa ser recomendada como a melhor, porém sugere-se que algum tipo de vigilância pós-alta seja realizada.

A escolha do método de vigilância, dependerá das condições de cada instituição considerando: tipo de gestão, tipo de clientela atendida, número de pessoal disponível no SCIH, estrutura física do hospital e funcionamento dos ambulatórios (se há retorno centralizado dos pacientes etc.), além do envolvimento técnico da equipe cirúrgica com o SCIH.

Estas considerações podem, ainda, serem analisadas sob o enfoque da importância da incidência da ISC, enquanto um indicador da qualidade do serviço prestado. Dado o contexto atual, onde o período de internação dos pacientes tem sido cada vez menor, com uma média de permanência de dois a três dias, os resultados deste estudo reforçam a importância do seguimento pós-alta para a detecção de ISC adicionais que vão se manifestar após a alta hospitalar.

E, caso este seguimento não seja realizado, não será permitido ao $\mathrm{SCIH}$ e à equipe cirúrgica conhecer a real incidência de ISC, impedindo assim que esforços sejam direcionados à redução desta tipo de infecção, que ocupa um lugar de destaque no contexto das infecções hospitalares com uma alta taxa de morbi-mortalidade.

\section{REFERÊNCIAS}

(1) Mangram AJ, Horan TC, Pearson ML, Silver LC, Jarvis WR. Guideline for prevention of surgical site infection. Am J Infect Control 1999, 27(2):97-132.

(2) Gaynes RP, Culver DH, Horan TC, Jonathan RE, Richards C, Tolson JS. Surgical site infection (SSI) rates in the Unites States, 19921998: The National Nosocomial Infections Surveillance System Basic SSI Risk Index. Clinical Infections Disease 2001; 33 (Suppl 2):S69-77.
(3) Grinbaum RS. Infecções do sitio cirúrgico e antibioticoprofilaxia em cirurgia. In: Rodrigues EAC, Mendonça JS, Amarante JMB, Alves Filho MB, Grinbaum RS, Richmann R. Infecções hospitalares: prevenção e controle. São Paulo: Sarvier; 1997. Pt 3, cap 2, p.149-61.

(4) Ferraz EM, Ferraz AB, Bacelar TS, Albuquerque HSTD, Vasconcelos MDMM, Leão CS. Controle de infecção em cirurgia do aparelho digestivo: resultado de um estudo prospectivo de 23 anos em 42.274 cirurgias. Rev Col Bras Cirur 2001; 28:17-25. 
(5) Horan TC, Gaynes RP, Martone WJ, Jarvis WR, Emori TG. CDC definitions of nosocomial surgical site infections, 1992: a modification of CDC definitions of surgical wound infections. Am J Infect Control 1992; 20(5):271-4.

(6) Martone WJ, Lee Nichols R. Recognition, prevention, surveillance and management of surgical site infections: introduction to the problem and symposium overview. Clin Infect Dis 2001; 33 (Suppl 2) S67-8.

(7) Starling CEF, Pinheiro SMC, Couto BRGM. Vigilância epidemiológica das infecções hospitalares na prática diária, ensaios. Belo Horizonte: Cutiara; 1993.

(8) Rabhae GN, Ribeiro Filho N, Fernandes AT. Infecção do sitio cirúrgico. In: Fernandes AT, editor. Infecções hospitalares e suas interfaces na área de saúde. São Paulo: Atheneu; 2000. p. 479-505.
(9) Oliveira AC. Controle de egresso cirúrgico: impacto na incidência da infecção de sítio cirúrgico em um hospital universitário. [dissertação]. Belo Horizonte (MG): Escola de Enfermagem da UFMG: 1999.

(10) Manian FA. Surveillance of surgical site infections in alternative settings: exploring the current options. Am J Infect Control 1997; 25(2):102-5.

(11) Couto RC, Pedrosa TMG, Nogueira JM. Infecção hospitalar: epidemiologia e controle. Rio de Janeiro: Medsi; 1997.

(12) Ferraz EM, Ferraz AAB, Coelho HSTA, Pereira VP, Sobral SML, Vasconcelos MDMM, Bacelar TS. Postdischarge surveillance for nosocomial infection: does judicious monitoring find cases? Am J Infect Control 1995; 23(5):290-4.
Infecção de sítio cirúrgico no seguimento pós-alta: impacto na incidência e avaliação dos métodos utilizados 